\title{
Design, Fabrication, and Testing of Active Skin Antenna with 3D Printing Array Framework
}

\author{
Jinzhu Zhou, ${ }^{1,2}$ Haitao Li, ${ }^{1}$ Le Kang, ${ }^{3}$ Baofu Tang, ${ }^{4}$ Jin Huang, ${ }^{1}$ and Zhiheng Cai ${ }^{1}$ \\ ${ }^{1}$ Key Laboratory of Electronic Equipment Structure Design, Ministry of Education, Xidian University, Xi'an 710071, China \\ ${ }^{2}$ State Key Laboratory of Structural Analysis for Industrial Equipment, Dalian University of Technology, Dalian 116024, China \\ ${ }^{3}$ National Key Laboratory of Antennas and Microwave Technology, Xidian University, Xi'an 710071, China \\ ${ }^{4}$ Nanjing Research Institute of Electronic Technology, Nanjing 210039, China
}

Correspondence should be addressed to Jinzhu Zhou; xidian_jzzhou@126.com

Received 25 August 2017; Accepted 17 October 2017; Published 23 November 2017

Academic Editor: Maggie Y. Chen

Copyright (c) 2017 Jinzhu Zhou et al. This is an open access article distributed under the Creative Commons Attribution License, which permits unrestricted use, distribution, and reproduction in any medium, provided the original work is properly cited.

\begin{abstract}
An active skin antenna with structural load-bearing and electromagnetic functions is usually installed in the structural surface of mobile vehicles such as aircrafts, warships, and high-speed train. This paper presents the design, fabrication, and testing of a novel active skin antenna which consists of an encapsulation shell, antenna skin, and RF and beam control circuits. The antenna skin which consists of the facesheet, honeycomb, array framework, and microstrip antenna elements was designed by using Bayesian optimization, in order to improve the design efficiency. An active skin antenna prototype with 32 microstrip antenna elements was fabricated by using a hybrid manufacturing method. In this method, 3D printing technology was applied to fabricate the array framework, and the different layers were bonded to form the final antenna skin by using traditional composite process. Some experimental testing was conducted, and the testing results validate the feasibility the proposed antenna skin structure. The proposed design and fabrication technique is suitable for the development of conformal load-bearing antenna or smart skin antenna installed in the structural surface of aircraft, warships, and armored vehicles.
\end{abstract}

\section{Introduction}

Over the last decade, structural, material, and antenna designers have collaborated to integrate the microstrip antennas (or planar spiral antennas) and microwave circuits into the structural surfaces of the aircraft, warship, and armored vehicles. This multidisciplinary effort has developed a new high payoff technique known as the skin antenna [1-3]. The skin antenna provides a new paradigm where the structural surface becomes an antenna, and it is not only a microwave device receiving or sending electromagnetic waves but also a load-bearing structure [2]. Compared with the antennas mounted on the structural surface, the skin antenna could have several advantages such as reducing the weight and improving the structural efficiency and the electromagnetic performance, and it will play increasingly a key role in future radar and wireless communications of mobile vehicles such as aircraft, high-speed train, car, and ship.
Many researchers have dedicated to the investigation of the skin antenna and proposed different concepts such as a conformal load-bearing antenna [4-6], structurally integrated antenna $[7,8]$, structurally embedded vascular antenna $[9,10]$, three-dimensionally integrated microstrip antenna [11-14], and composite antenna [15-17]. Almost all the investigations have been dedicated to the design and fabrication of the passive skin antenna. The basic structure of the passive skin antenna is a sandwich construction consisting of factsheets, honeycomb core, microstrip or spiral antenna elements, and feeding networks. The antenna elements and feeding networks are inserted into the factsheets and honeycomb, and the epoxy adhesive is applied to bond the different components. However, in practice, the active skin antenna is usually required for radar and wireless communication applications. Different from the passive skin antennas, the active skin antenna has a steerable beam pointing capability, and its structure is more complicated. Generally, the low temperature co-fired ceramics (LTCC) 
and printing circuit board (PCB) are utilized to fabricate the active skin antenna. For example, in [3], LTCC was applied to fabricate the RF layer (containing antenna elements, feeding networks, low-noise amplifiers, phase shifters, and power amplifiers) of the active skin antenna. In [2], the substrate Rogers 5880 was applied to fabricate the antenna array embedded in the skin structure. However, for a large skin antenna, it is costly and different to fabricate the antenna array using the LTCC and PCB. In addition, the conventional fabrication of the skin antenna is the lack of the flexible use and reuse of the antenna elements, due to the fixedness of the antenna elements in the antenna array.

In recent years, $3 \mathrm{D}$ printing (also called additive manufacturing) was applied to fabricate the antenna and microwave devices [18]. Compared to conventional manufacturing techniques, 3D printing offers several advantages, including the capability of a more flexible design, less prototyping time, cost reduction, much less human interaction, and a faster product development cycle [19]. 3D printing technology has been investigated to realize advanced wireless devices, such as antennas, from $\mathrm{GHz}$ to $\mathrm{THz}$ frequency range. Antennas with different structures such as horn antennas [20], patch antennas [21], conformal antennas [22], gradient index lens antennas [23], and reflect array antennas [24] have been realized using 3D printing techniques. The $3 \mathrm{D}$ printed passive circuits and components for higher frequencies have also been demonstrated. In [25], dielectric ridge waveguides, a photonic crystal filter, and a power splitter were all printed on a commercially available $3 \mathrm{D}$ printer. Almost all the investigations above have been dedicated to the design and fabrication of the $3 \mathrm{D}$ printed passive antenna and microwave devices. As for the active antenna array, Chen and her teams have firstly demonstrated an inkjet-printed flexible phasedarray antenna without any lithography process [26-28]. Passive and active components, such as microstrip transmission lines, phase shifters, and RF power distribution networks, are all developed adopting a room temperature 3D printing process. The electronics, such as switch and amplifiers, are printed with ultrapure CNT solution. Their proposed design and fabrication technology show great advantages for developing the smart skin antenna, electronic paper, epidermal electron, and so on.

In this paper, 3D printing techniques were applied to fabricate the array framework into which microstrip antenna elements were embedded for radiating electromagnetic waves. Subsequently, composite process was applied to bond the array framework, the facesheet, honeycomb, and the antenna elements to form the final antenna skin which can provide the structural load-bearing and electromagnetic receiving sending functions simultaneously. The motivation of this investigation is to provide the design and fabrication technology of a new active skin antenna structure for wireless communications of mobile vehicles such as aircraft, highspeed train, car, and ship. Compared with the active skin antenna in $[2,3]$, the proposed antenna has the following advantages such as low cost, easy maintenance, and flexible use and reuse of antenna elements. In addition, the active skin antenna prototype with 32 microstrip antenna elements was fabricated by using a hybrid manufacturing method. In this method, 3D printing technology was applied to fabricate the array framework, which can simplify the manufacturing process and reduce the cost of the antenna skin, especially for the complex conformal array framework. Subsequently, the different layers were bonded to form the final antenna skin by using traditional composite process.

This paper presents the design, fabrication, and testing of a new active skin antenna with 3D printing array framework. This proposed antenna consists of an encapsulation shell, an antenna skin, and RF and beam control circuits. Bayesian optimization was applied to design the antenna skin, in order to improve the design efficiency. The active skin antenna prototype with 32 microstrip antenna elements was fabricated, and some experiments were conducted to evaluate the fabricated antenna prototype. The remainder of this paper is organized as follows. The overall structure of the active skin antenna is presented in Section 2. Section 3 describes the design of an active skin antenna prototype, and Bayesian optimization is applied to obtain an optimal design of the antenna skin. The fabrication of the active skin antenna prototype is succinctly described in Section 4. Section 5 presents the testing results of the skin antenna prototype. Finally, Section 6 concludes the paper.

\section{Overall Structure of Active Skin Antenna}

An active skin antenna can provide the structural loadbearing and electromagnetic receiving sending functions simultaneously. In the following, we present a novel active skin antenna structure. Figure 1(a) shows an application of the distributed active skin antenna installed in the wing of an aircraft, and the overall structural configuration of the active skin antenna is shown in Figure 1(b).

From Figure 1(b), it is observed that the proposed antenna consists of an encapsulation shell, an antenna skin, and RF (i.e., transmitting and receiving circuits) and beam control circuits. The encapsulation shell is filled with the RF and beam control circuits. Bolt connections are applied to connect the encapsulation shell and load-bearing framework which is a part of the wing. The encapsulation shell can protect the internal circuits against the external loads such as impact loads and aerodynamic loads. The antenna skin can provide the structural load-bearing and electromagnetic receiving functions, and it is composed of an antenna layer and a protective layer which contains the facesheet and honeycomb/foam. The antenna skin is embedded into the load-bearing framework using adhesive bonding or bolt connection technology. The RF circuits comprise feeding networks, low-noise amplifiers, phase shifters, and power amplifiers, and so on. The beam control circuits consist of direct current (DC) power and control circuits, and it generates the beamforming signal and supplies the power required by the RF circuits. Every antenna element in the antenna skin is fed by a SMA which connects the RF circuits by a flexible RF cable. 


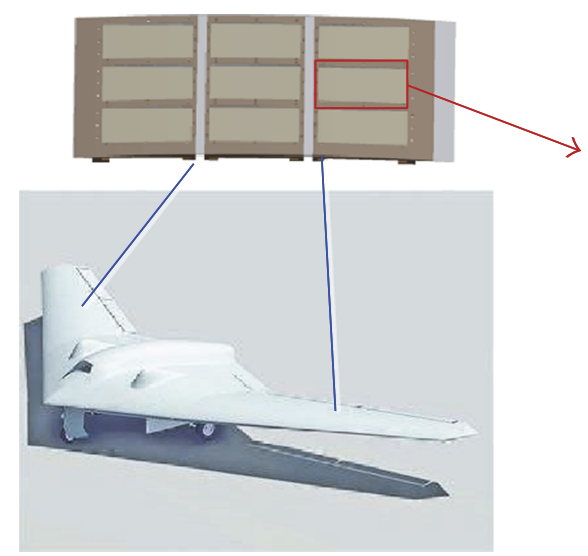

(a)

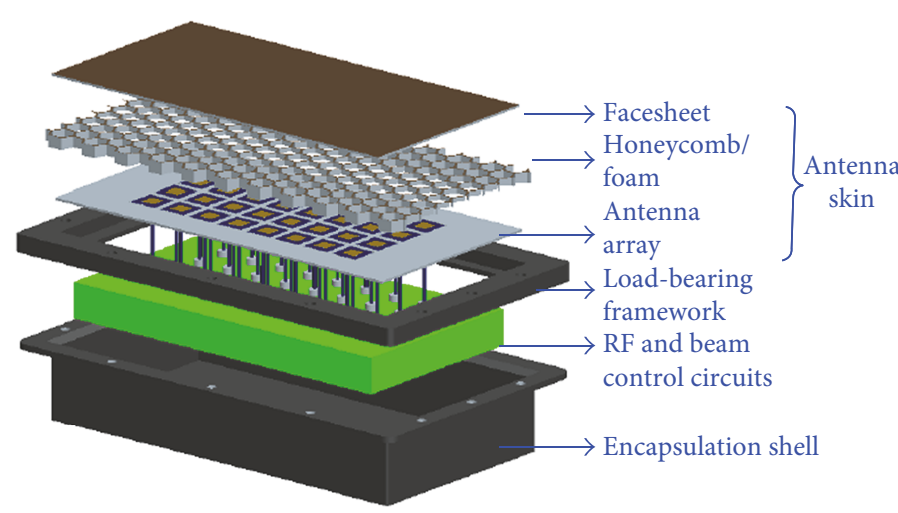

(b)

Figure 1: Schematic diagram of active skin antenna. (a) Application of active skin antenna. (b) Structural configuration of active skin antenna.

\section{Design of Active Skin Antenna Prototype}

According to the antenna structure in Figure 1, this section presents the design of an active skin antenna prototype with only the RF receiving function. The desired performance requirements are listed in Table 1 . In this table, the desired deformation displacement and structural stress mean the maximum allowable structural stiffness and strength, when a $50 \mathrm{~N}$ load is applied to the central point of the antenna structure. In the following, the antenna skin is designed using Bayesian optimization, and then the RF receiving and beam control circuits are described concisely.

3.1. Bayesian Optimal Design of Antenna Skin. Figure 2 shows the structural configuration of the proposed antenna skin. From Figure 2, it is observed that the antenna skin comprises the facesheet, honeycomb, and antenna array. The facesheet, honeycomb, and antenna array are bonded to form the final antenna skin. In the antenna skin, the antenna array consists of 32 microstrip antenna elements and an array framework fabricated by using 3D printing technology, and every antenna element is embedded into the array framework using the adhesive bonding technology. Table 2 shows the materials which every layer needs and the electrical properties of these materials.

Figure 3 presents the flowchart of the antenna skin design. As shown in Figure 3, the antenna skin design consists of two steps. The first step is to design the length, width of the antenna element, and the position of the feed port. The second step is to optimize the thickness of facesheet and honeycomb in this skin antenna, and an electromechanical codesign optimization is proposed to balance the mechanical and electrical requirements.

3.1.1. Formulation of Optimizing Antenna Elements. The first stage is to specify the substrate materials, antenna element type, and the dimensions of the antenna element substrate, according to some predefined performances. In the antenna skin prototype, the length $L_{\mathrm{g}}$ and width $W_{\mathrm{g}}$ of the antenna element substrate were $28 \mathrm{~mm}$, as shown in Figure 4 .
TABle 1: Performance requirements of the skin antenna.

\begin{tabular}{lcc}
\hline Layer & Performance specifications & Value \\
\hline \multirow{2}{*}{ Electrical performance } & Central frequency & $5.8 \mathrm{GHz}$ \\
& $S_{11}$ of element & $\leq-20 \mathrm{~dB}$ \\
& Antenna array gain & $\geq 18 \mathrm{~dB}$ \\
\hline \multirow{2}{*}{ Mechanical performance } & Structural stress & $\leq 240 \mathrm{MPa}$ \\
& Deformation displacement & $\leq 12 \mathrm{~mm}$ \\
\hline
\end{tabular}

Utilizing the initial design determined by some empirical formulas, the simulation model of the microstrip antenna element was developed using HFSS VBScript. In order to obtain an optimal design, the paper constructed the following optimization model:

$$
\begin{array}{ll}
\text { Find } & \mathbf{x}=\left[x_{1}, x_{2}, D x, D y\right], \\
\text { Min } & S_{11}=S_{0}-\max \left[0, S_{0}-S\right], \\
& \left|f-f_{0}\right|<\varepsilon, \\
\text { s.t. } & \mathbf{x}_{l}<\mathbf{x}<\mathbf{x}_{u},
\end{array}
$$

where the design variables $x_{1}$ and $x_{2}$ represent the length and width of the microstrip antenna elements, respectively. $D x$ and $D y$ represent the $x$-axis and $y$-axis coordinates of the feed port, respectively; $S_{11}$ is the return loss; $S$ is the return loss under the current design size; $S_{0}$ is upper bound of the return loss; $f_{0}$ is the center frequency of antenna element; $\varepsilon$ is the resonant frequency deviation from the allowable value; and $\mathbf{x}_{1}$ and $\mathbf{x}_{\text {up }}$ denote the lower bound and upper bounds of the design variable $\mathbf{x}$, respectively.

As for the antenna element design used in the antenna skin prototype, the parameters $S_{0}, \varepsilon$, and $f_{0}$ were chosen as $-20 \mathrm{~dB}, 0.05 \mathrm{GHz}$, and $5.8 \mathrm{GHz}$, respectively. The lower bound $\mathbf{x}_{1}$ and upper bounds $\mathbf{x}_{\mathrm{up}}$ are $[10,10,-3.2,-3.2]$ and $[15,15,3.2,3.2]$, respectively.

3.1.2. Electromechanical Codesign Formulation. The aim in the second stage is to obtain optimal dimensions of the 


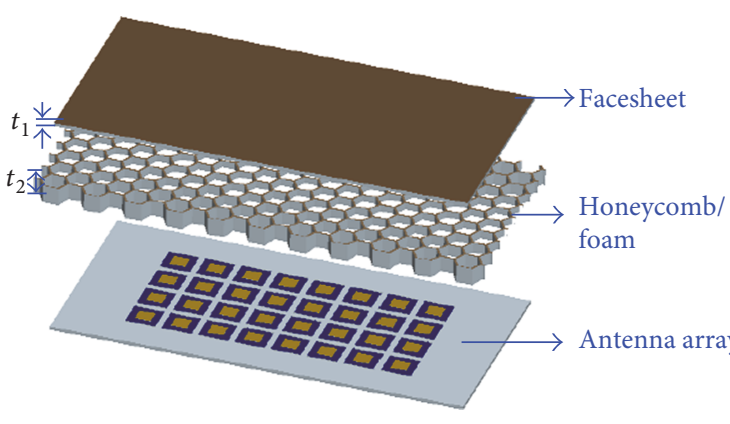

Structure of antenna skin

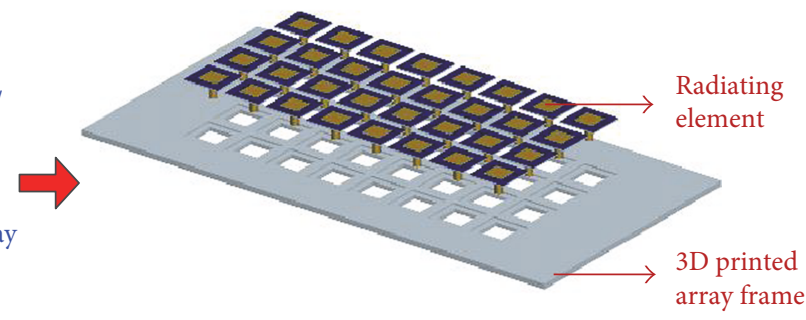

Structure of antenna array

FIGURE 2: Structural configuration of antenna skin.

TABLE 2: Electrical properties of every layer.

\begin{tabular}{lcc}
\hline Layer & Material & Dielectric constant \\
\hline Upper facesheet & Glass/epoxy & 4 \\
Honeycomb core & Nomex honeycomb & 1.1 \\
Array framework & Photosensitive resin & 2.4 \\
Antenna element & RO4350B & 3.66 \\
\hline
\end{tabular}

facesheet and honeycomb core, which influence the mechanical and electrical performance. The mechanical performance consists of the structural weight, stiffness, and strength, which can be calculated by the mechanical model of the antenna structure. The electrical performance such as the radiation pattern can be obtained from an electromagnetic simulation model.

In this stage, the antenna array was firstly constructed by utilizing the optimal antenna element. Subsequently, the electromagnetic model of the antenna skin was developed using HFSS VBScript, and the mechanical model of the skin antenna structure was developed using ANSYS parametric design language. Finally, the following electromechanical codesign formulation was proposed to determine the thickness of the facesheet and honeycomb which can simultaneously meet the mechanical and electrical requirements.

$$
\begin{array}{ll}
\text { Find } & \mathbf{d}=\left[t_{1}, t_{2}\right]^{\mathrm{T}} \\
\text { Min } & G=- \text { Gain }(\mathbf{d}) \\
& \left|f_{i}-f_{0}\right|<e, \quad i=1,2, \ldots, 32 \\
& S_{i}<S_{1}, \quad i=1,2, \ldots, 32 \\
\text { s.t. } & \delta_{\max }(\mathbf{d}) \leq[\delta] \\
& \sigma_{\max }(\mathbf{d}) \leq[\sigma] \\
& \mathbf{d}_{1} \leq \mathbf{d} \leq \mathbf{d}_{\mathrm{h}}
\end{array}
$$

where the design variable $\mathbf{d}=\left[t_{1}, t_{2}\right]^{\mathrm{T}}$ will be solved by the optimization; $t_{1}$ and $t_{2}$ represent the thickness of facesheet and honeycomb, as shown in Figure 2. The antenna gain $G$ ain $(\mathbf{d})$ of the antenna skin is used as the optimization objective, and $[\delta]$ and $[\sigma]$ are the allowable deformation displacement and structural stress, respectively. $\delta_{\max }(\mathbf{d})$ and $\sigma_{\max }(\mathbf{d})$ are the maximum deformation displacement and maximum structural stress under the current design variable $\mathbf{d}$, respectively. In this paper, the allowable maximum deformation displacement $\delta_{\max }(\mathbf{d})$ and maximum structural stress $\sigma_{\max }(\mathbf{d})$ are $12 \mathrm{~mm}$ and $240 \mathrm{MPa}$, respectively. $\mathbf{d}_{1}$ and $\mathbf{d}_{\mathrm{h}}$ are the predefined lower bound and upper bound, respectively.

The mechanical performances $\boldsymbol{\delta}_{\max }(\mathbf{d})$ and $\sigma_{\max }(\mathbf{d})$ are determined using the following mechanical model of the antenna skin structure:

$$
\mathbf{K}(\mathbf{d}) \boldsymbol{\delta}=\mathbf{F},
$$

where $\mathbf{K}(\mathbf{d})$ is the stiffness matrix and it is a function of the design variable $\mathbf{d}$. $\mathbf{F}$ is the vector of the load. $\delta$ is the displacement vector of the node deformation.

In this paper, the maximum deformation displacement $\delta_{\max }(\mathbf{d})$ and maximum structural stress $\sigma_{\max }(\mathbf{d})$ are used to evaluate the mechanical stiffness and strength before the antenna structure is destroyed. The maximum displacement $\delta_{\max }(\mathbf{d})$ is defined as the deflection at the central point of the antenna skin, when a predefined load is applied to the antenna structure.

3.1.3. Solution Based on Bayesian Optimization. Bayesian optimization is a statistical framework that uses information gained from past experiments to model and minimize an arbitrary objective function, and it works by building and querying cheap surrogate models which estimate the behavior of real objective functions which are expensive to evaluate [29]. Surrogate models are typically built using Gaussian process regression (GPR). GPR is to fit previously observed data and used to make predictions about the objective values in areas not yet explored. These predictions are easy to compute and can intelligently choose the next set of parameters such that solutions are found with a minimal number of expensive objective function.

In this subsection, the objective function in (1) and (2) was built by GPR model with given training data $(\mathbf{X}, \mathbf{Y})=$ $\left\{\mathbf{x}_{i}, \mathbf{y}_{i}\right\}_{i=1}^{N}$. According to the research in [29-31], the new point $\mathbf{x}_{N+1}$ is obtained by a search algorithm, and the new 


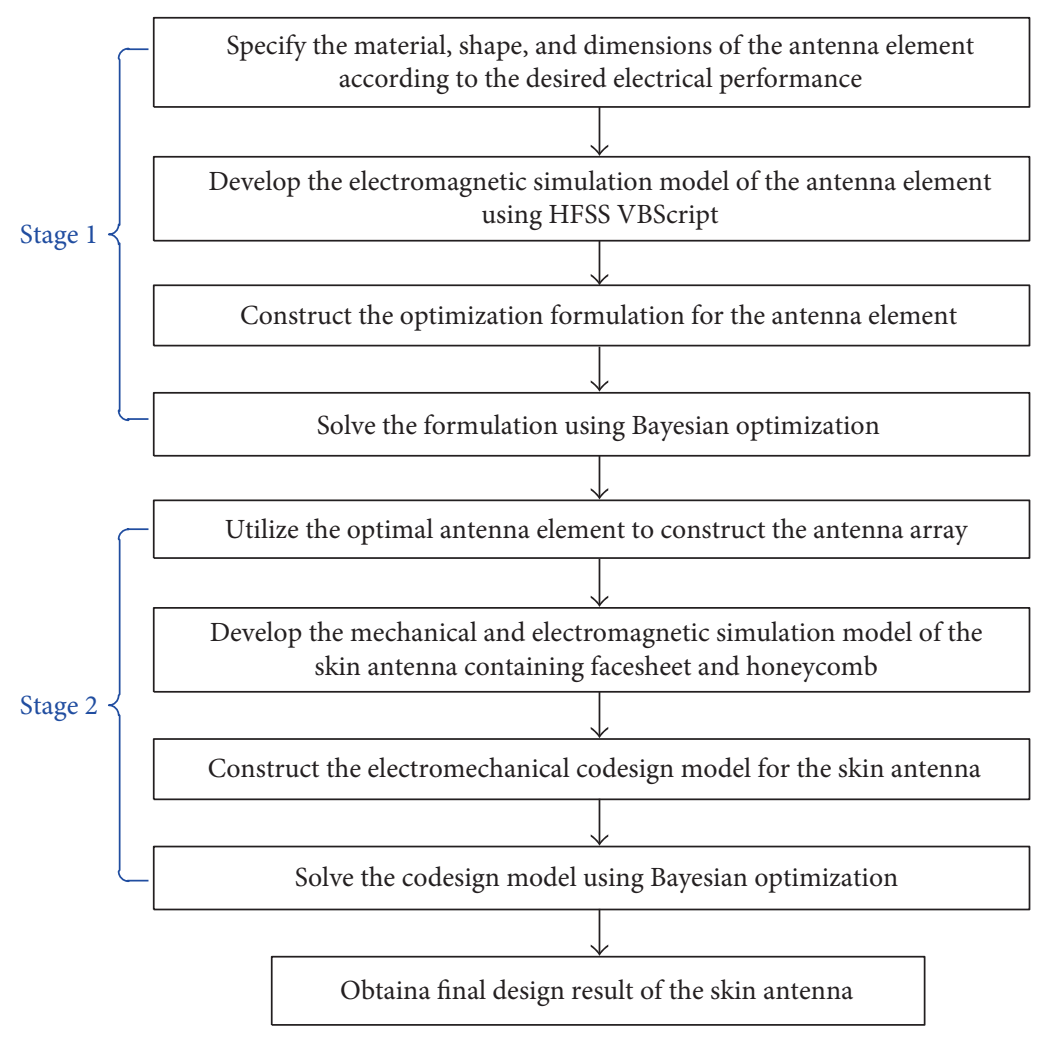

Figure 3: Flowchart of the antenna skin design.

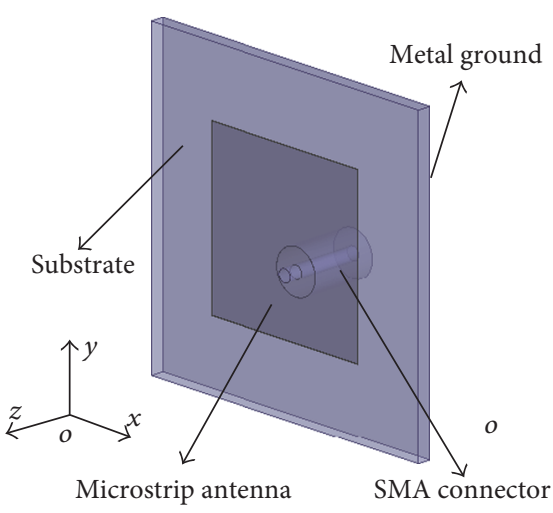

Side view

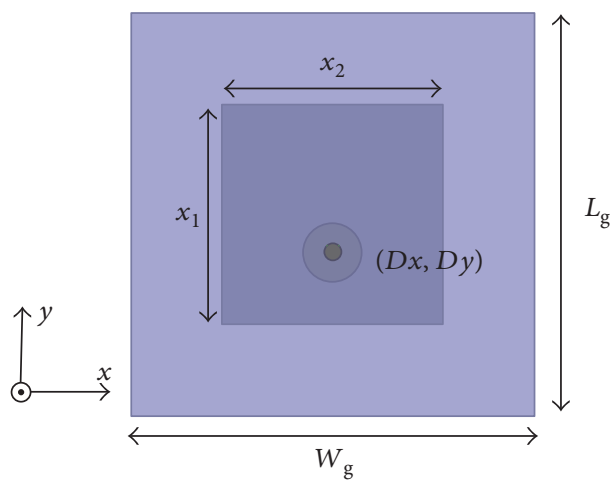

Top view

FIGURE 4: Geometry of microstrip antenna element.

output, $\mathbf{y}_{N+1}$, can be expressed with the predicted mean $m\left(\mathbf{x}_{N+1}\right)$ and the standard deviation $\sigma\left(\mathbf{x}_{N+1}\right)$ as

$$
\begin{aligned}
\mu\left(\mathbf{x}_{N+1}\right) & =\mathbf{k}^{\mathrm{T}}\left[\mathbf{K}+\sigma_{N}^{2} \mathbf{I}\right]^{-1} \mathbf{Y}, \\
\sigma^{2}\left(\mathbf{x}_{N+1}\right) & =\mathbf{k}\left(\mathbf{x}_{N+1}, \mathbf{x}_{N+1}\right)-\mathbf{k}^{\mathrm{T}}\left[\mathbf{K}+\sigma_{N}^{2} \mathbf{I}\right]^{-1} \mathbf{k}, \\
\mathbf{y}_{N+1} & =\mu\left(x_{N+1}\right) \pm \sigma\left(x_{N+1}\right),
\end{aligned}
$$

where $N$ is the number of training data, $\mathbf{I}$ is the identity matrix, $\mathbf{K}$ denotes the kernel matrix for the training data $(\mathbf{X}, \mathbf{Y}), \mathbf{k}$ represents the covariance matrix between $\mathbf{x}_{N+1}$ and $\mathbf{X}=\left(\mathbf{x}_{1}, \mathbf{x}_{2}, \ldots, \mathbf{x}_{N}\right), \mathbf{k}\left(\mathbf{x}_{N+1}, \mathbf{x}_{N+1}\right)$ signifies the selfcovariance matrix, and $\sigma_{N}$ is the noise deviation and the coefficient.

This GPR model provides a posterior distribution of the unknown function. We can choose the next value of the function representing the targeted values by minimizing an acquisition function. In this paper, the lower confidence bound (LCB) is used as the acquisition function to find the optimal value of the design variables [29], and it is expressed as follows:

$$
\mathbf{x}_{N+1}=\arg \min _{i}\left[\mu\left(x_{i}\right)-\kappa \sigma\left(x_{i}\right)\right]
$$




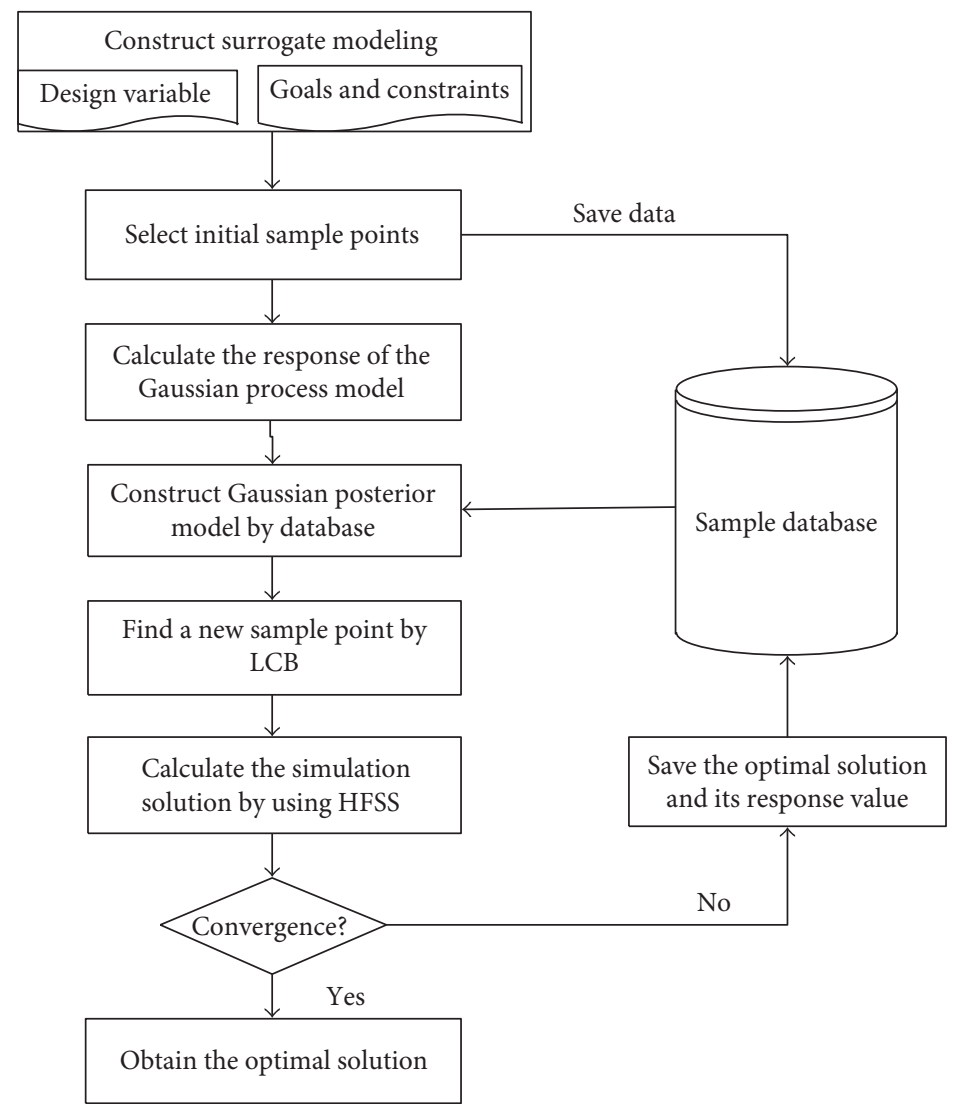

FIGURE 5: Flowchart of Bayesian optimization solution.

where $\kappa>0$ and $\kappa=\left(2 \log \pi^{2} x^{2} / 12 v\right)^{0.5}$ (where $v=0.05$ ); $\mu\left(x_{i}\right)$ and $\sigma\left(x_{i}\right)$ are determined from (4) and (5).

The Bayesian optimization above was applied to solve the optimization model in (1) and (2). The objective functions were built using GPR. Figure 5 presents the flowchart of Bayesian optimization solution, and the optimal value of the design variable is obtained by using the following procedure.

(1) Determine design variables and design space and build the target model which is to be optimized. The initial sample points are selected by the Latin hypercube sampling method in the whole design space. Let $K=1$, which represents the number of iteration.

(2) Fit a Gaussian distribution model by calculating the response of the Gaussian process model based on the multivariate normal distribution at sampling points.

(3) Optimize LCB acquisition function to find out the next design point $x_{t}$.

(4) Perform an electromagnetic simulation at $x_{t}$ by using HFSS to get the actual response value.

(5) If the response obtained in the previous step meets the convergence, stop the procedure; otherwise, go to the step 6 .
(6) Add the point sampled in step 3 to the database and return to step 2 .

It is important to note that the selection of the next sample does not require the computation of electromagnetic model developed by HFSS, since (7) is computed only based on the previous simulation results, which minimizes computational time. Unlike most optimization techniques, this approach provides a posterior distribution of the unknown function and hence the search involves determining the function (rather than the output itself) that is closer to the targeted goal.

\subsubsection{Design Results}

\section{(1) Optimization Results of Antenna Element}

In this part, the antenna element was optimized according to the optimization model in (1) by using the Bayesian optimization algorithm and genetic algorithm. Figures 6(a) and 6(b) show the optimization iterative process of the Bayesian algorithm and genetic algorithm, respectively. Compared with the two figures, it is found that Bayesian optimization needs 16 iterations to obtain the target solution which satisfies the design requirement. However, the genetic algorithm needs 50 iterations to achieve the target solution. The comparison of iteration numbers shows that the genetic algorithm is more likely to fall into the local optimal solution than the Bayesian algorithm. Table 3 shows the 


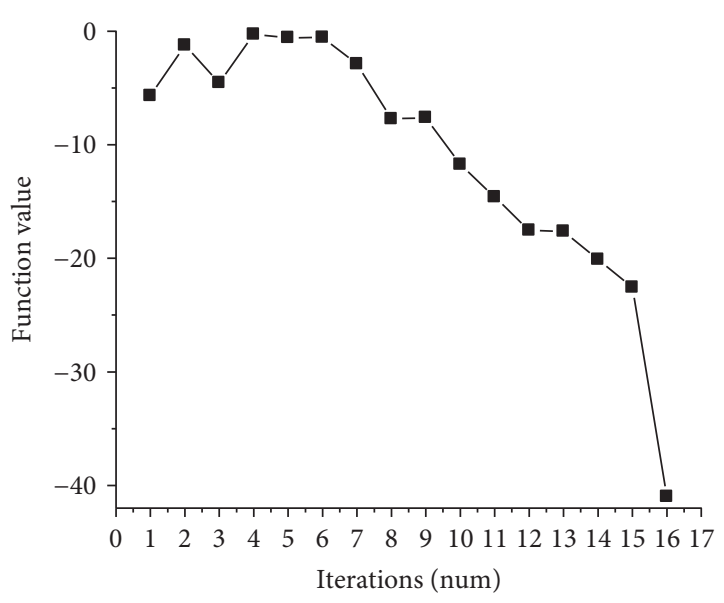

(a) Bayesian algorithm iteration

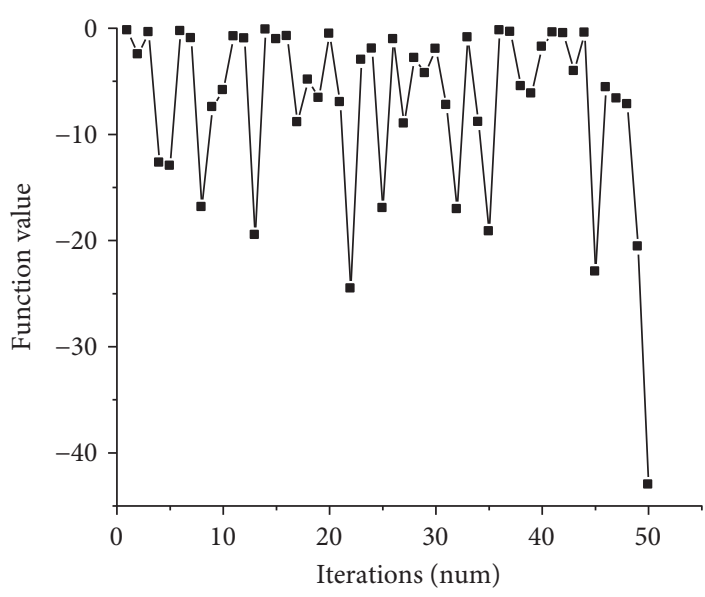

(b) Genetic algorithm iteration

FIGURE 6: Iteration process of the Bayesian algorithm and genetic algorithm.

TABLE 3: Comparison of the antenna element optimization results.

\begin{tabular}{lccccc}
\hline & $\begin{array}{c}\text { Function valuation } \\
\text { numbers }\end{array}$ & Time consuming (s) & Function value (dB) & Center frequency (GHz) & Target solution (mm) \\
\hline Bayesian algorithm & 17 & 1521 & -41 & 5.808 & {$[12.9,12.9,0,0]$} \\
Genetic algorithm & 50 & 3209 & -43 & 5.763 & {$[13,13,-0.09,0]$} \\
\hline
\end{tabular}

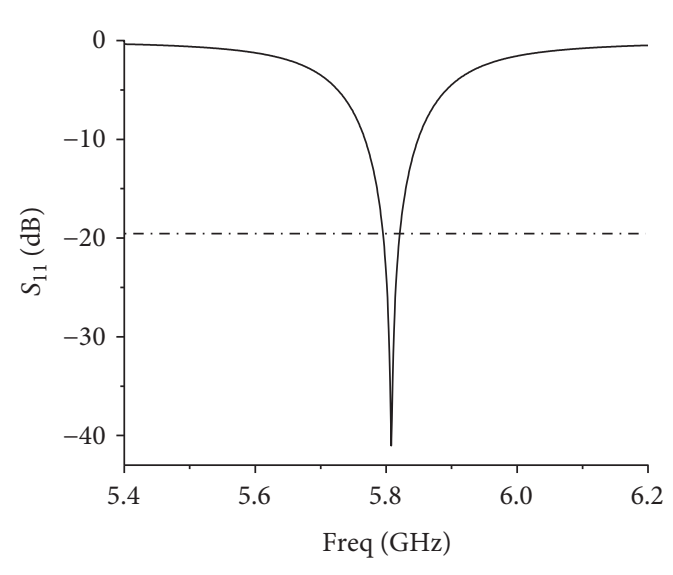

(a) Return loss

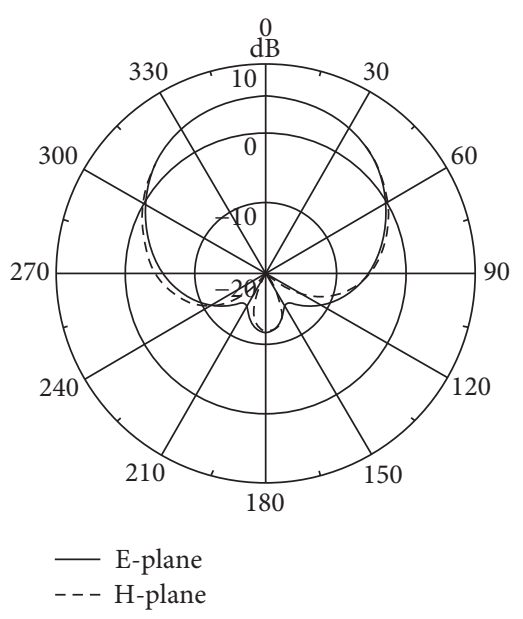

(b) Radiation pattern of microstrip antenna

element

FIGURE 7: Simulated return loss and radiation pattern of the radiating element.

comparing results of the two algorithms for optimizing the antenna element. As seen from these results, the target solutions obtained by the Bayesian optimization algorithm and genetic algorithm are similar. However, the center frequency obtained by the Bayesian algorithm is much closer to $5.8 \mathrm{GHz}$. Besides, the time required by the Bayesian algorithm is less than half the time required by the genetic algorithm. Figure 7 shows the optimal return loss and the gain pattern of the antenna element obtained by Bayesian optimization. From Figure 7(b), it is observed that the gain of the antenna element is about $6.23 \mathrm{dBi}$.

\section{(2) Optimization Results of Antenna Skin}

In this investigation, the antenna array was firstly constructed by utilizing the optimal antenna element from the previous section. Subsequently, according to (2), the thickness of facesheet and honeycomb was optimized by the Bayesian algorithm and genetic algorithm, respectively. 
TABLE 4: Comparison of the antenna skin optimization results.

\begin{tabular}{lccc}
\hline & $\begin{array}{c}\text { Time } \\
\text { consuming } \\
(\mathrm{min})\end{array}$ & $\begin{array}{c}\text { Function } \\
\text { value }(\mathrm{dB})\end{array}$ & $\begin{array}{c}\text { Target } \\
\text { solution } \\
(\mathrm{mm})\end{array}$ \\
\hline Bayesian algorithm & 191.4 & 21.4 & {$[0.27,2]$} \\
Genetic algorithm & 429 & 21.5 & {$[0.26,4.25]$} \\
\hline
\end{tabular}

Table 4 provides some comparisons of the two algorithms. As shown in Table 4, the calculating time required by the two algorithms is different. The time required by the genetic algorithm is more than twice time required by the Bayesian algorithm. Therefore, it is clear that the Bayesian algorithm is much faster than the genetic algorithm. Utilizing the optimization results from the Bayesian algorithm, Figure 8 shows the simulation results of the antenna skin gain, and its gain is $21.4 \mathrm{~dB}$. When a $50 \mathrm{~N}$ load is applied to the central point of the antenna skin structure, the mechanical performance of the antenna skin is shown in Figure 9. The maximum deformation displacement is $11.9 \mathrm{~mm}$, and the maximum structural stress is $225 \mathrm{MPa}$. These results can meet the desired performance requirements in Table 1.

3.2. Design of RF Receiving and Beam Control Circuits. The $\mathrm{RF}$ receiving and beam control circuits are responsible for the reception of electromagnetic waves, amplitude, and phase control of antenna elements. Figure 10 presents the block diagram of the circuit in the active skin antenna prototype. The circuit consists of the same four RF receiver modules and a beam control circuit.

From Figure 10, it is observed that each RF receiver module connects eight microstrip antenna elements by using RF cables. Each RF receiver module consists of an active receiving circuit and two-stage feed networks. Figure 11 shows the internal circuits in every RF receiver module. Each active receiving circuit has eight channels which realize the connection between the antenna element and the first-stage feed network. Each receiving channel consists of a lownoise amplifier (LNA), a 6-bit digital phase shifter, a variable attenuator, and a gain amplifier. In this prototype, the LNA and gain amplifier utilize the same chip PMA3-83LN+ manufactured by Mini-Circuits. The voltage-controlled variable attenuator HMC712LP3C is manufactured by $\mathrm{ADI}$, and the 6-bit digital phase shifter MAPS-011008 is from MACOM. The attenuator and phase shifter are used to control the amplitude and phase, respectively, of incoming signal on each individual channel, and the control commands of the attenuator and phase shifter are from the beam control circuits, as shown in Figure 10.

In this paper, two-stage feed networks are used to combine each channel signal received by the antenna element to the RF terminal. The feed networks were designed by using integrated circuit combiners and distributed microstrip transmission line. The integrated circuit combiner WP4A+ and SCN-2-65+ from Mini-Circuits Corporation were used to realize the combination of four or two equal amplitude and phase power signals, respectively. The Wilkinson feed networks realized by distributed transmission line were designed to connect the output channel and the integrated

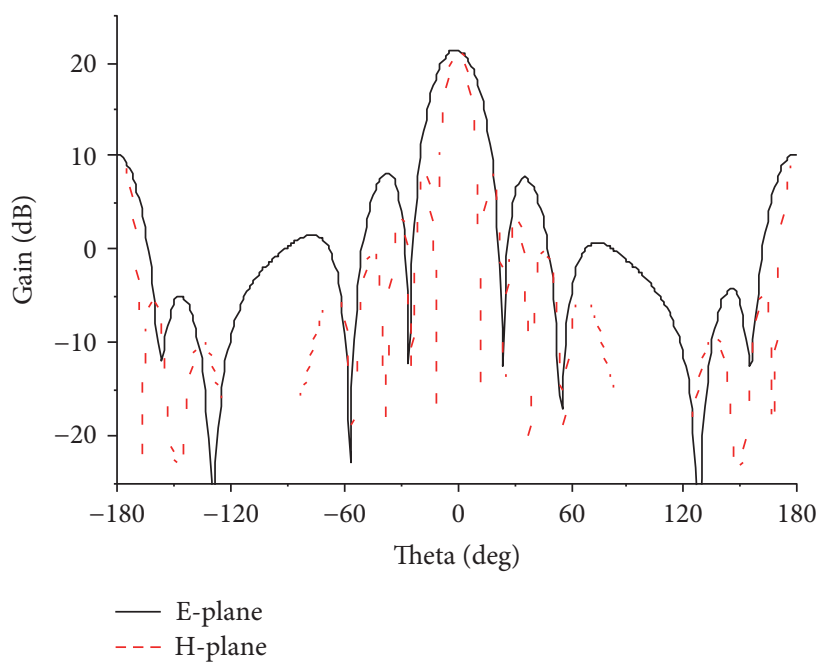

FIGURE 8: Simulation result of antenna skin gain.

circuit combiner chip. Figure 12 presents the design of the first-stage feed networks (i.e., 4:1 combining networks), due to the limitation of the length of the paper. From the simulation results in Figure 12(b), it is found that the network can obtain approximately equal insertion loss and equal phase characteristics in the $5.4-6.2 \mathrm{GHz}$ frequency range.

\section{Fabrication of Active Skin Antenna}

This section presents the fabrication of an active skin antenna prototype, and the fabrication process is succinctly described in the following.

The antenna skin was fabricated by using a hybrid manufacturing approach. In this paper, the hybrid manufacturing approach refers to the combination of $3 \mathrm{D}$ printing manufacturing and traditional manufacturing process. The $3 \mathrm{D}$ printing manufacturing was applied to fabricate the antenna array framework, and the remainder was fabricated by using traditional manufacturing process. The hybrid manufacturing approach can simplify the manufacturing process and reduce the cost of the antenna skin, especially for the complex conformal array framework.

First, every layer in the antenna skin was manufactured separately, according to the design results above. During the course of the fabrication, the antenna array framework was fabricated by using the 3D printing technology, and 32 microstrip antenna elements were fabricated by screen printing methods. Figure 13(a) presents the fabricated microstrip antenna elements and array framework. Subsequently, the microstrip antenna elements were embedded into the array framework using the adhesive bonding technology, as shown in Figure 13(b). The designed facesheet and honeycomb were prepared by confirming their mechanical and electrical performances. For the facesheet, two-ply glass/ epoxy prepregs were symmetrically laid up, and each layer was bonded on the top or bottom of another one in the design sequence by using the epoxy film adhesive. Subsequently, the layers were aligned and laminated according to the assembly sequence of each layer. After being covered 


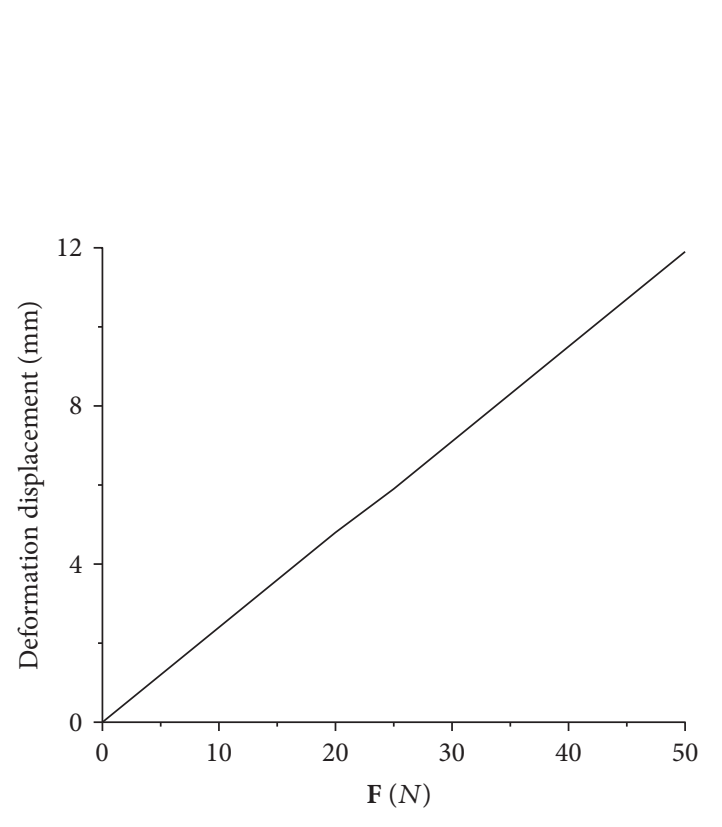

(a) Load-displacement curves

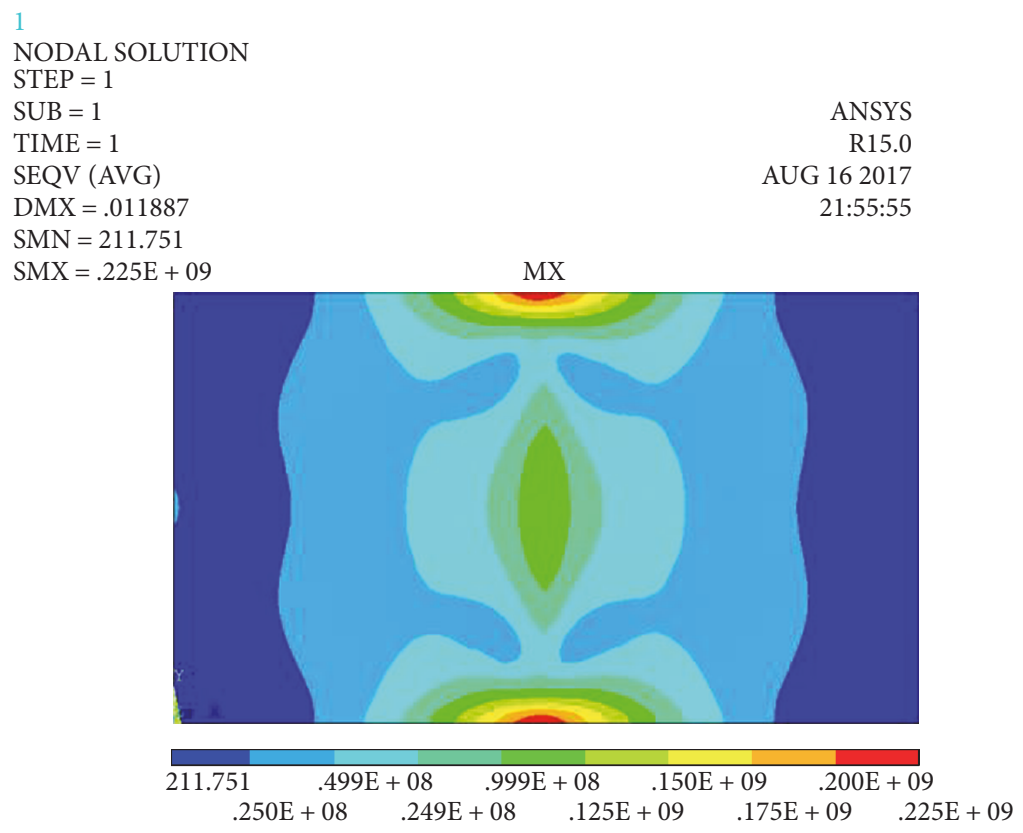

(b) Stress cloud of mechanical model

FigURE 9: Analysis results of mechanical performance.

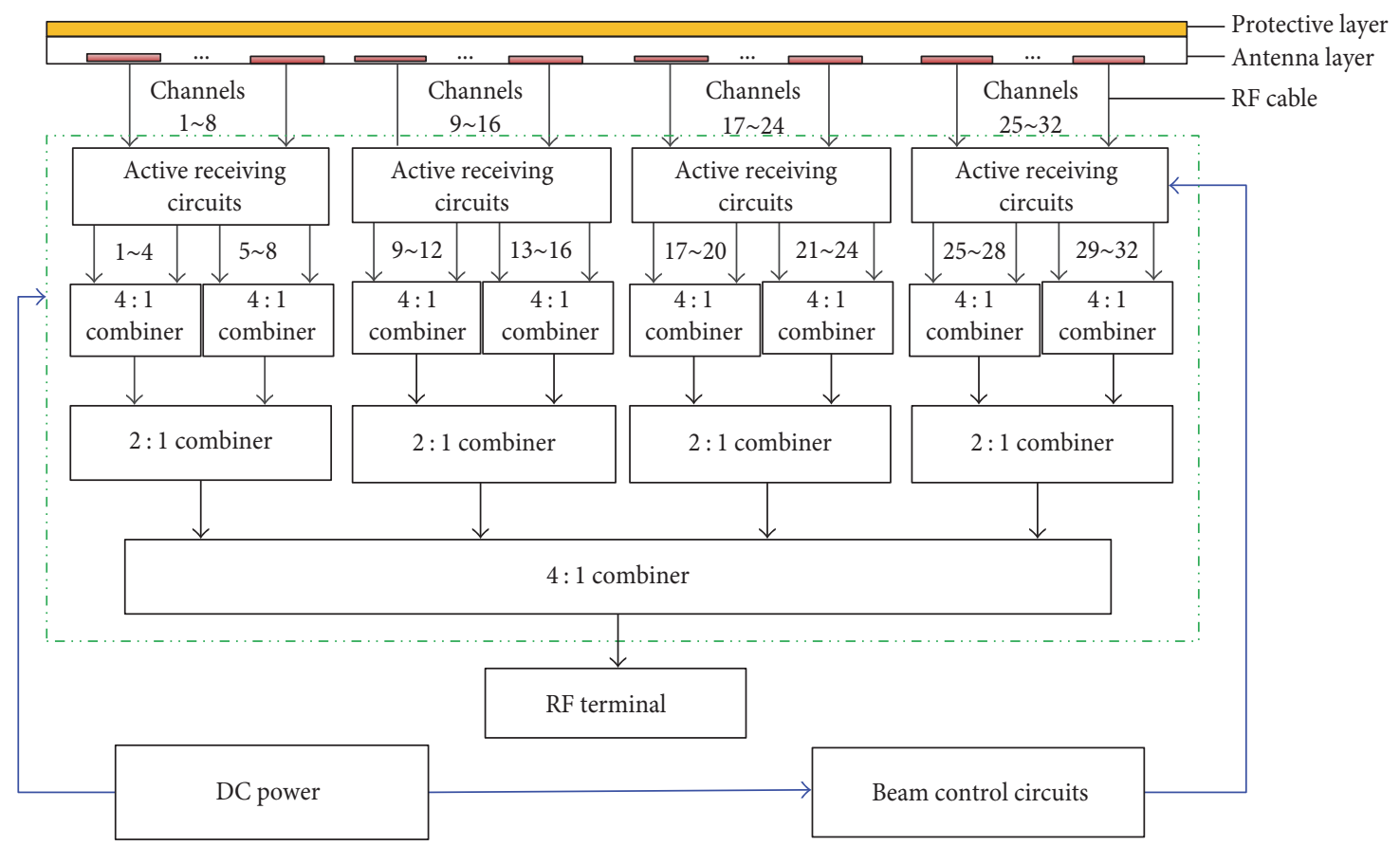

FIGURE 10: Block diagram of information transmission of smart skin antenna prototype.

by a vacuum bag, the assembly was cocured in an autoclave, according to the recommended curing cycle for this adhesive $\left(125^{\circ} \mathrm{C}\right.$ for $180 \mathrm{~min}$ at a pressure of $\left.0.45 \mathrm{MPa}\right)$. Figure 13(f) shows the final skin antenna sample. The $\mathrm{RF}$ receiving and beam control circuits were fabricated by printing circuit board technology. Figure 14 shows the fabricated active skin antenna prototype. The soft RF label was utilized to connect the antenna skin with the RF receiving and beam control circuits.

\section{Testing Results}

Utilizing the fabricated antenna prototype, we have conducted some experiments to validate the proposed design and fabricating method. Figure 15 presents the experimental system which consists of a test fixture, active skin antenna, and near-field measuring system. Moreover, the absorbing wave materials cover the metal surface of the test fixture to avoid the reflection and scattering of electromagnetic wave. 


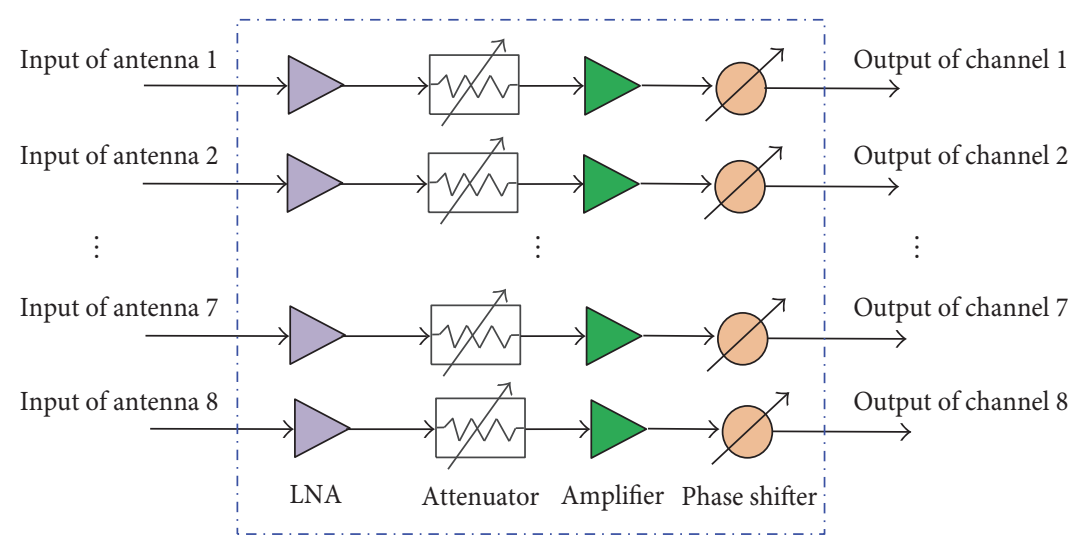

Figure 11: Active receiving circuits in a RF receiver module.

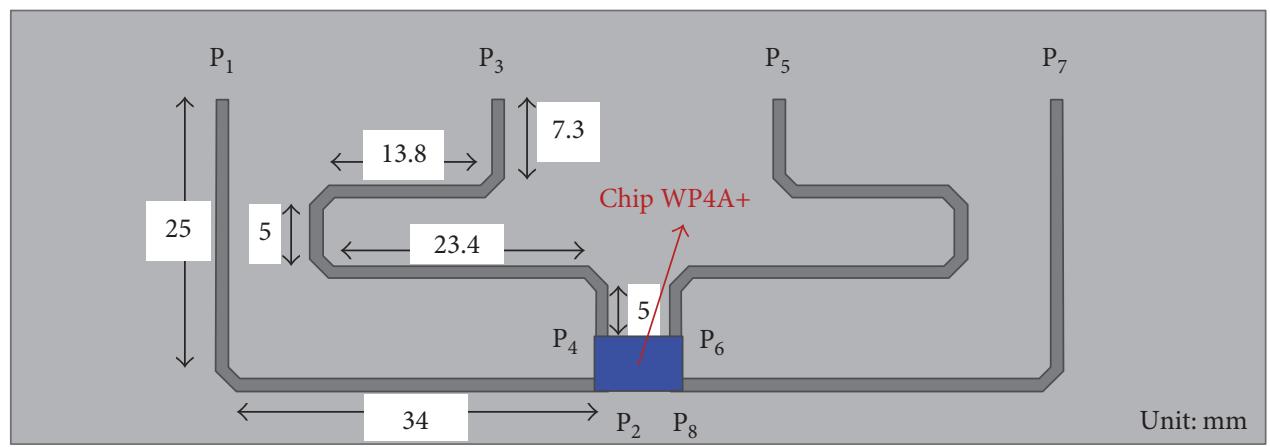

(a) 4:1 combining network
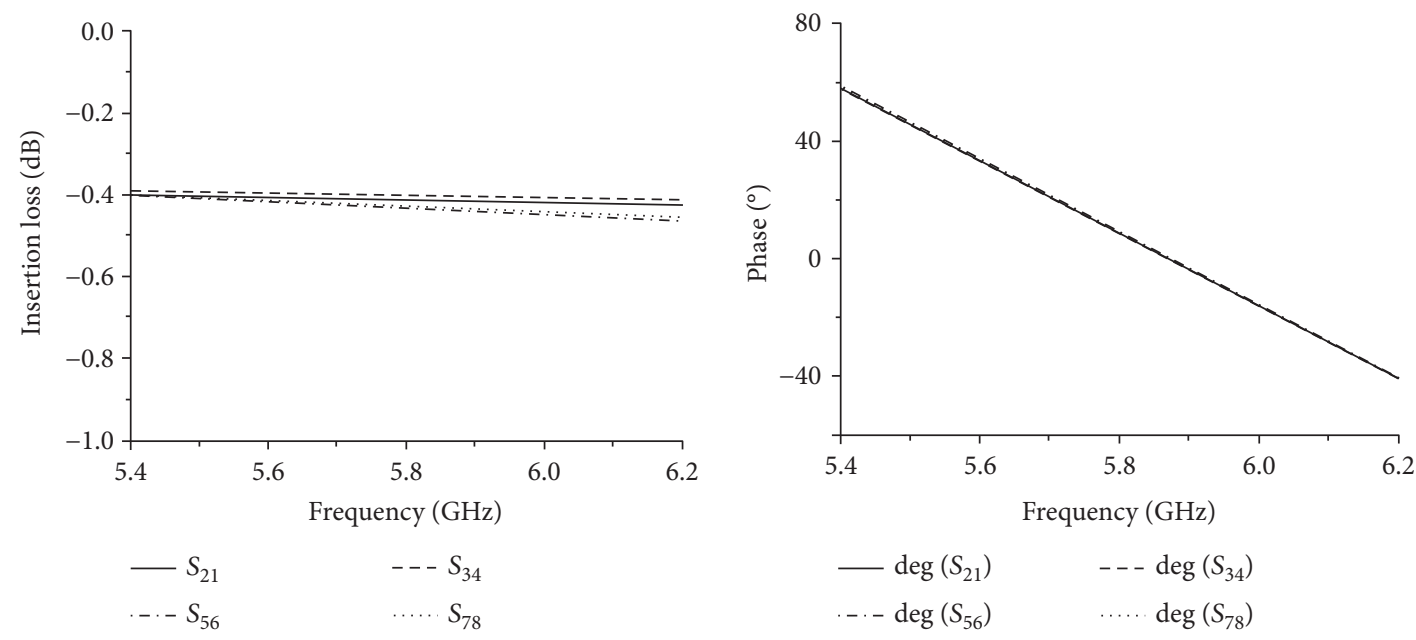

(b) Insertion loss and phase of microstrip line

Figure 12: Design results of $4: 1$ combining networks.

The experimental results and discussions were presented in the following.

5.1. Comparisons of Simulation and Measured Results. In order to validate the Bayesian optimal design, this paper firstly presents the measured return loss of microstrip antenna element and then presents the comparisons of measured and simulation radiation patterns of the antenna prototype at $5.8 \mathrm{GHz}$. Figure 16 gives the measured return loss of the 32 fabricated antenna elements. It is found that the measured return loss satisfies the design specification in Table 1. The maximum error between the simulation and measured center frequency is $0.03 \mathrm{GHz}$. Figure 17 presents the comparisons between the measured and simulation radiation patterns of the antenna prototype at $5.8 \mathrm{GHz}$. The maximum gain of the active skin antenna is about $21.2 \mathrm{dBi}$.

From Figure 17, it is also found that the measured radiation patterns are closed to the simulation ones. However, 


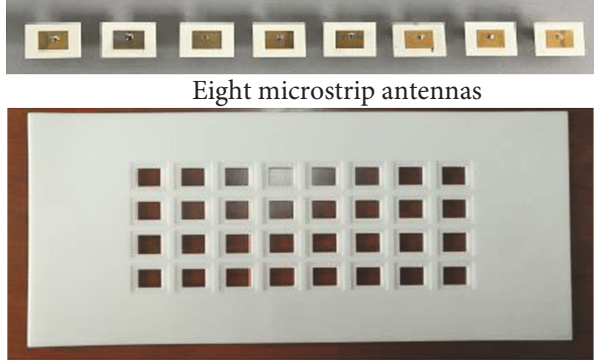

Antenna array frame fabricated by $3 \mathrm{D}$ printed technology

(a) Preparation of antenna elements and framework

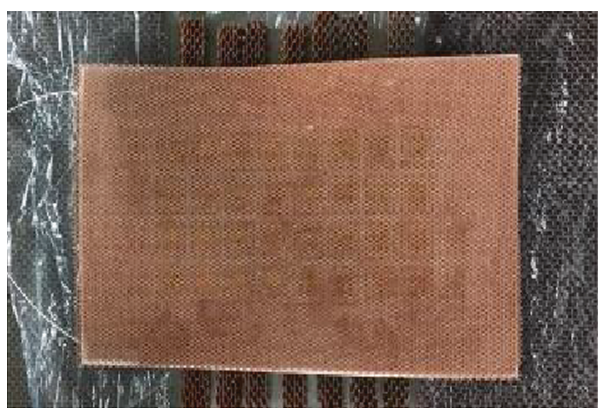

(c) Preparation of facesheet and honeycomb

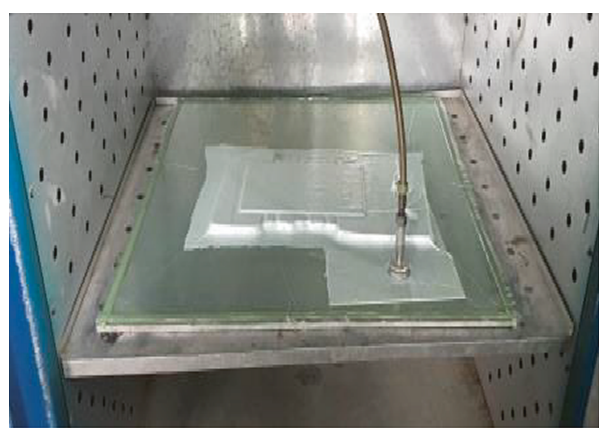

(e) Cocured process after coved by a vacuum bag

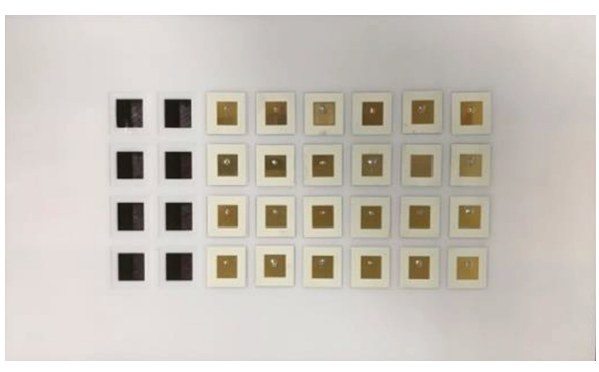

(b) Embedding antenna elements into framework

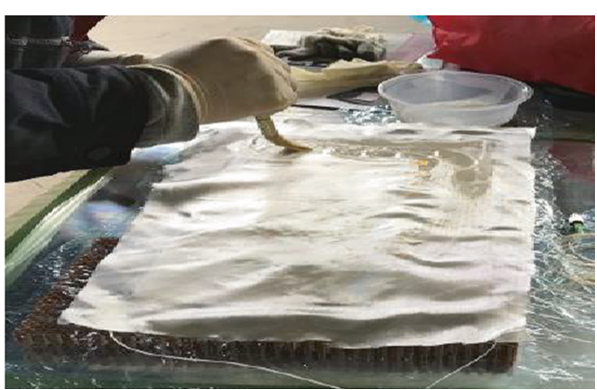

(d) Coating adhesive after the assembly

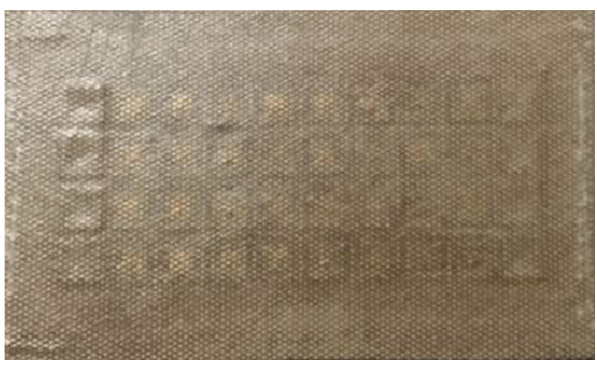

(f) Multifunctional antenna skin specimens

FIGURE 13: Fabrication process of antenna skin.

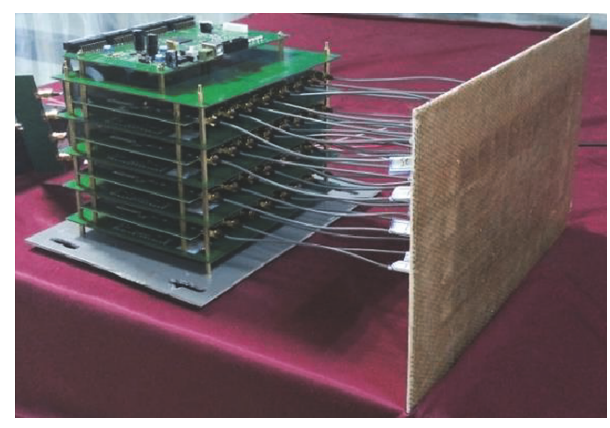

Figure 14: Active skin antenna prototype.

there is a little difference between the measured and simulation side lobe. One of the reasons for this is the effect of the adhesive in the skin antenna. The adhesive that bonds the different layers to provide mechanical strength also influences the electrical performance of the skin antenna. The adhesive influences the effective dielectric constant in the

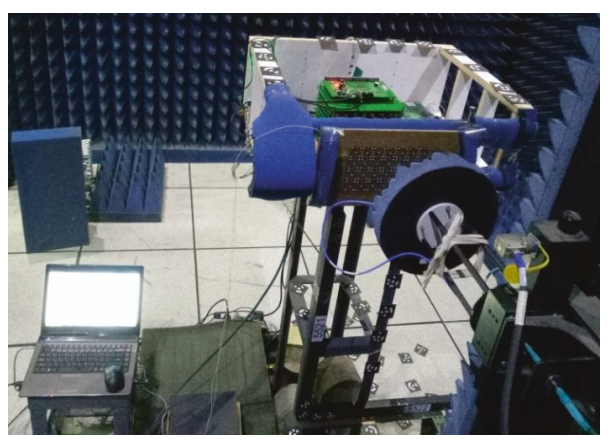

FIgURE 15: Measurements of active skin antenna prototype.

antenna structure. The change of the effective dielectric constant leads to inaccurate simulation results and makes the central frequency have a drift. In addition, the manufacturing errors in RF receiving circuits and microstrip antenna elements, which mean the structural dimension deviations 


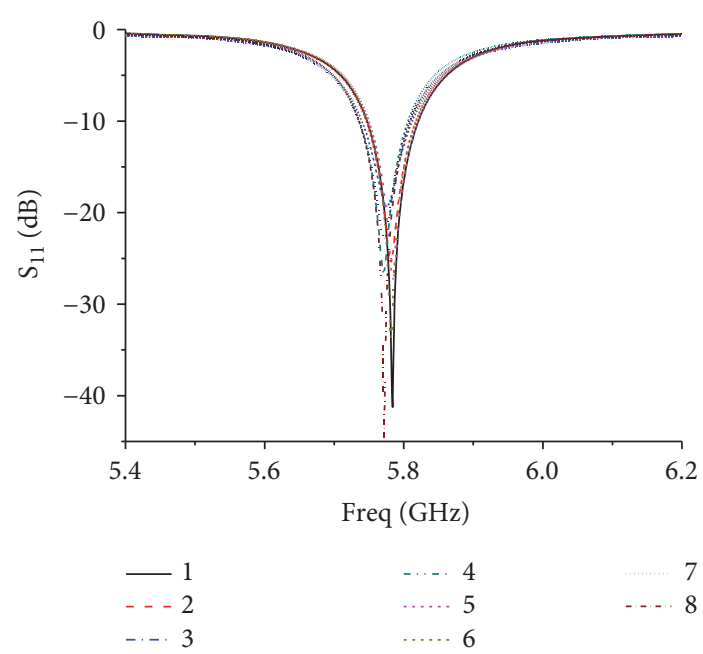

(a) $S_{11}$ of antenna element 1 to antenna element 8

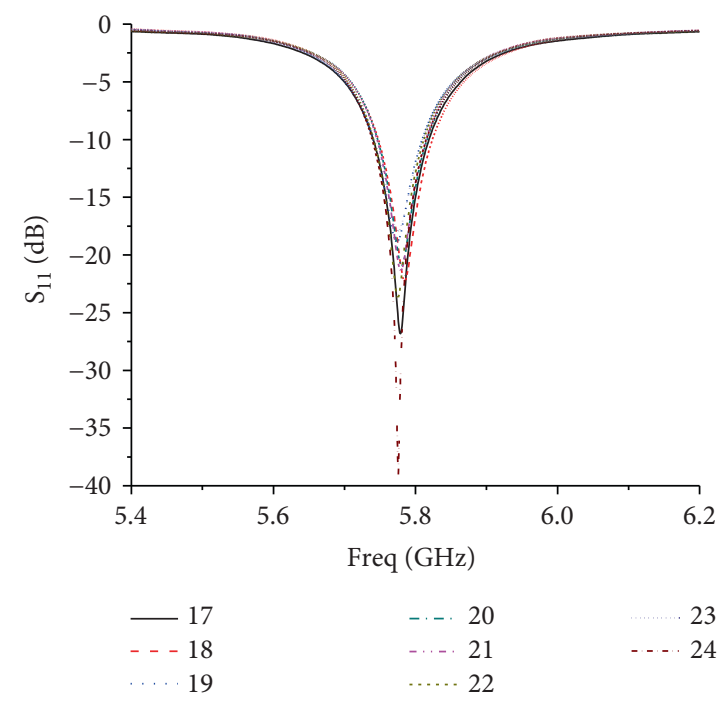

(c) $S_{11}$ of antenna element 16 to antenna element 24

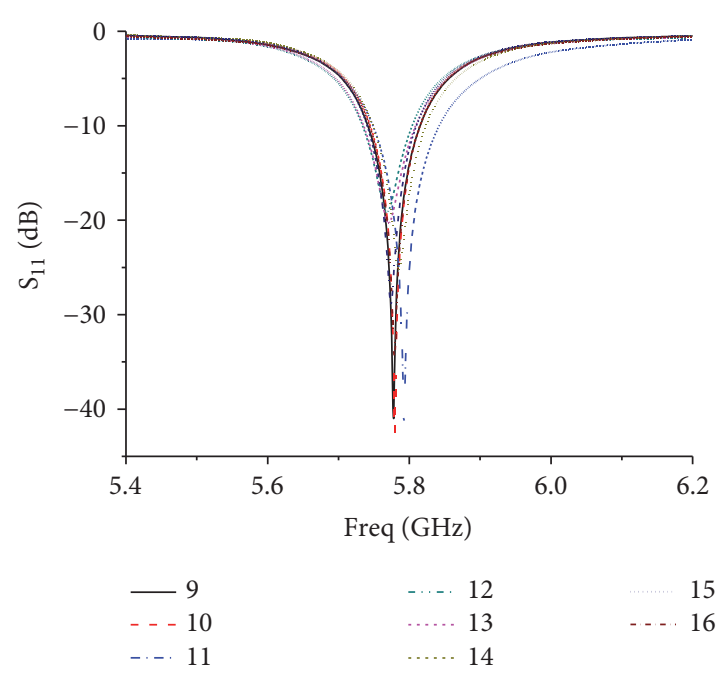

(b) $S_{11}$ of antenna element 9 to antenna element 16

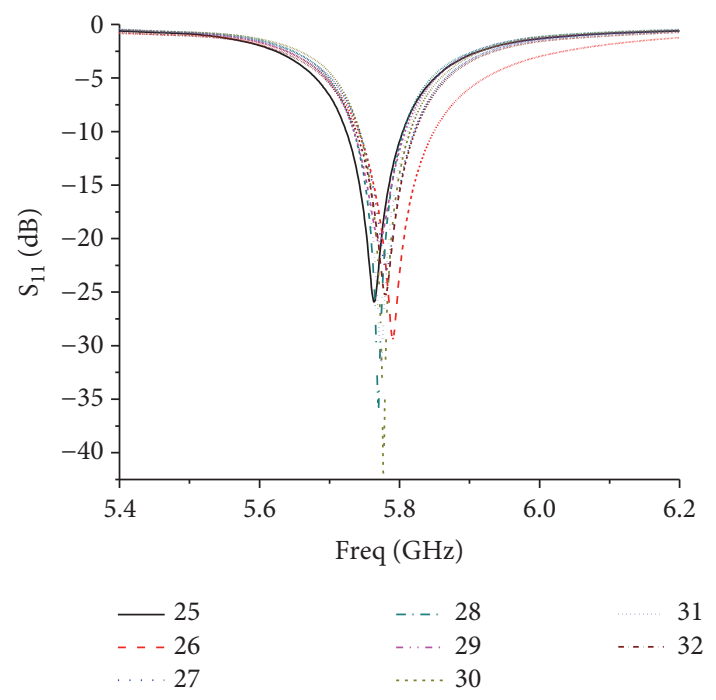

(d) $S_{11}$ of antenna element 24 to antenna element 32

Figure 16: Measured return loss.

relative to the optimal design dimensions, also lead to the differences between the simulation and measured results of the radiation patterns and return loss.

5.2. Measured Scanning Patterns. To demonstrate the electromagnetic performance, the scanning radiation characteristics of the fabricated antenna prototype were also measured in an anechoic chamber. Figure 18 presents the measured scanning patterns in xoz-plane and yoz-plane of the active skin antenna operating at $5.8 \mathrm{GHz}$. It is observed that the active skin antenna has a peak gain of $21.2 \mathrm{dBi}$ and the side lobe levels (SLL) in xoz-plane and yoz-plane are about -14.1 and $-13.7 \mathrm{~dB}$, respectively. In addition, it is observed that the scanning range in xoz-plane is from $-29.0^{\circ}$ to $29.3^{\circ}$ and that the scanning range in yoz-plane is from $-14.2^{\circ}$ to $14.9^{\circ}$. Table 5 gives some electrical performance indexes extracted from the measured scanning patterns in Figure 18. From the table, it is found that there is some difference between the desired beam direction and actual beam direction. This difference may be due to the imperfections of the measurement system such as the phase error caused by the manufacturing errors in RF receiving circuits.

\section{Conclusions}

This paper presents a novel active skin antenna structure which consists of an encapsulation shell, an antenna skin, and RF and beam control circuits. The antenna skin comprises facesheet, honeycomb, microstrip antenna elements and an array framework fabricated by 3D Printing technology. The different layers were bonded to form the final antenna skin. In addition, Bayesian optimization method was applied to design the microstrip antenna element and the thickness of facesheet and honeycomb of the skin antenna, in order to obtain an optimal design and improve the design efficiency. The active skin antenna prototype with 32 microstrip antenna elements was fabricated and some 


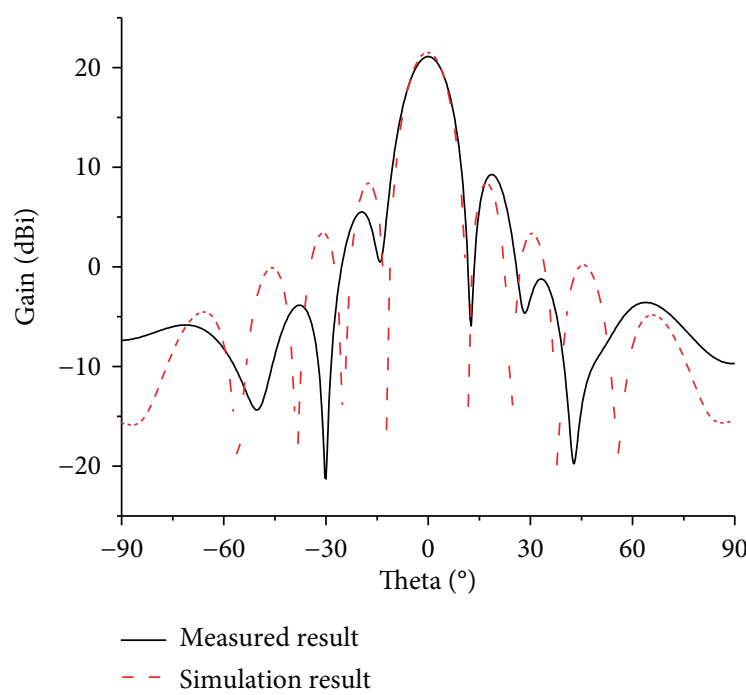

(a) xoz-plane

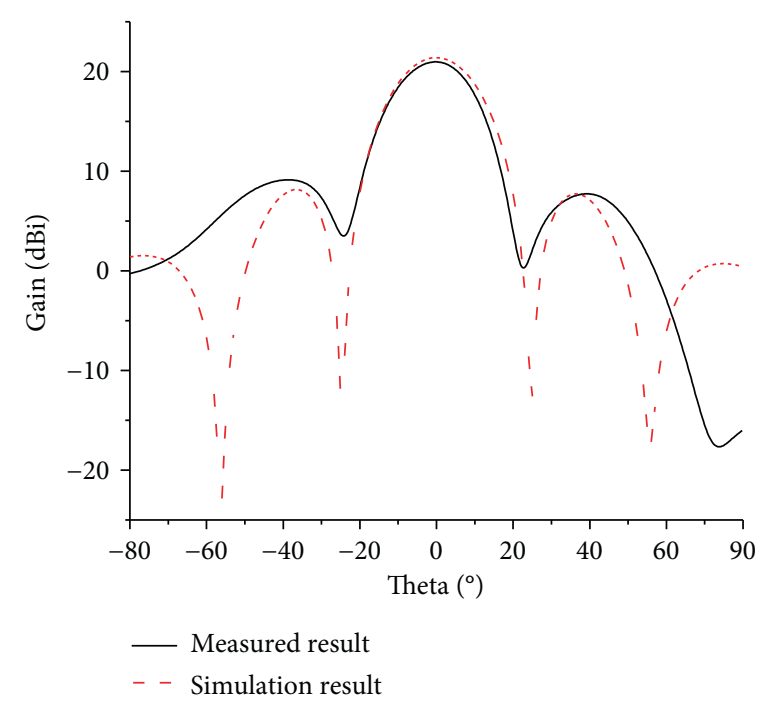

(b) yoz-plane

FIGURE 17: Comparisons of the measured and simulation radiation patterns of the prototype at $5.8 \mathrm{GHz}$.

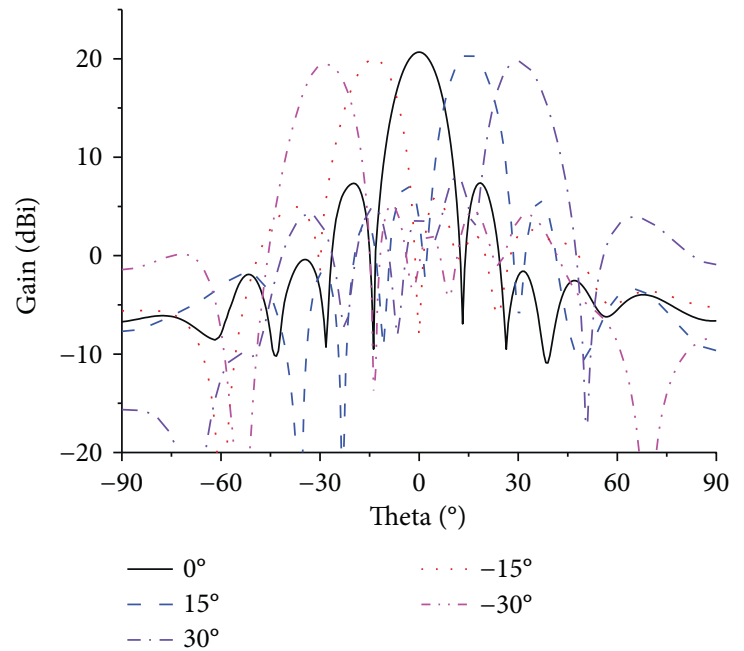

(a) xoz-plane

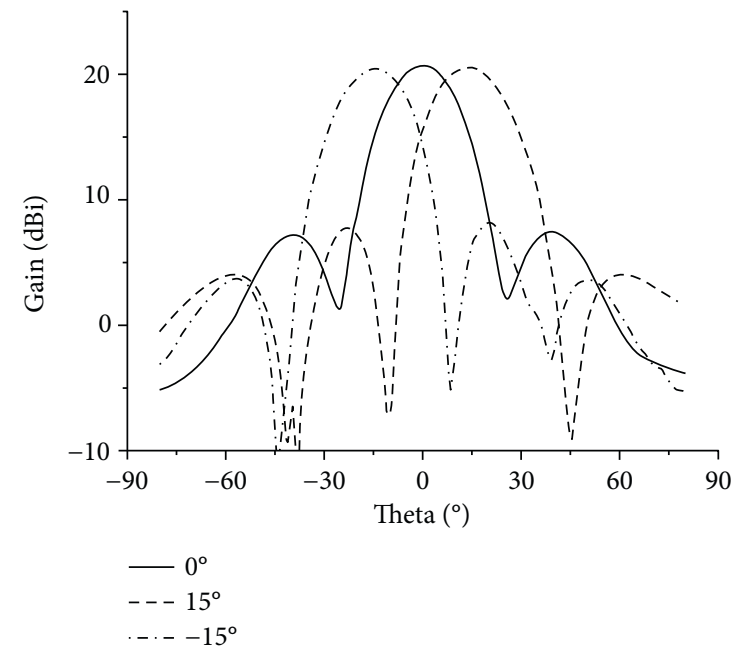

(b) yoz-plane

FIGURE 18: Measured scanning patterns of the active skin antenna prototype at $5.8 \mathrm{GHz}$.

TABLE 5: Electrical performance indexes of active skin antenna under undeformed conditions.

\begin{tabular}{lccccc}
\hline Plane & Desired beam direction $\left(^{\circ}\right)$ & Actual beam direction $\left(^{\circ}\right)$ & Gain $(\mathrm{dB})$ & Beam width $\left(^{\circ}\right)$ & Maximum side lobe level $(\mathrm{dB})$ \\
\hline xoz-plane & $0^{\circ}$ & 0.2 & 21.2 & 11.6 & -14.1 \\
xoz-plane & $15^{\circ}$ & 14.7 & 20.9 & 12.2 & -13.5 \\
xoz-plane & $30^{\circ}$ & 29.3 & 20.2 & 13 & -12.1 \\
xoz-plane & $-15^{\circ}$ & -14.3 & 20.8 & 12.5 & -13.8 \\
xoz-plane & $-30^{\circ}$ & -29.0 & 19.9 & 13.8 & -11.3 \\
yoz-plane & $0^{\circ}$ & 0.4 & 21.2 & 21.2 & -13.7 \\
yoz-plane & $15^{\circ}$ & 14.9 & 21.0 & 23 & -12.5 \\
yoz-plane & $-15^{\circ}$ & -14.2 & 20.9 & 23.8 & -12.6 \\
\hline
\end{tabular}

experiments were conducted to evaluate the fabricated antenna prototype. The comparisons between the simulation and measured results show that Bayesian optimization method can obtain accurate design results under the less computing time. The measured scanning radiation patterns validate the feasibility the proposed antenna skin structure. 
Our design and fabrication technique is suitable for the development of conformal load-bearing antenna or smart skin antenna installed in the structural surface of aircraft, warships, and armored vehicles. Furthermore, Bayesian optimization method provides an alternative approach to realize the fast and accurate optimal design of antenna and microwave circuits.

\section{Conflicts of Interest}

The authors declare that they have no conflicts of interest.

\section{Acknowledgments}

This project is supported by the National Natural Science Foundation of China (Program no. 51775405, no. 51490664, no. 51575419, and no. 51305323), Natural Science Basic Research Plan in Shaanxi Province of China (Program no. 2016JM5002), Opening Foundation of State Key Laboratory of Structural Analysis for Industrial Equipment (Program no. GZ15110), the Fundamental Research Funds for the Central Universities (Grant no. JB160420), and Defense Basic Research Program (Program no. JCKY2016210B002). Zhaoxi Liu and Haiyang Li from Xidian University provided experimental help during the project. Their efforts were crucial to the completion of the research.

\section{References}

[1] J. Kim, J. Y. Jang, G. H. Ryu, J. H. Choi, and M. S. Kim, "Structural design and development of multiband aerovehicle smart skin antenna," Journal of Intelligent Material Systems and Structures, vol. 25, pp. 631-639, 2014.

[2] S. H. Son, S. Y. Eom, and W. B. Hwang, "Development of a smart-skin phased array system with a honeycomb sandwich microstrip antenna," Smart Materials and Structures, vol. 17, article 035012, 2008.

[3] J. Zhou, J. Huang, Q. He, B. Tang, and L. Song, "Development and coupling analysis of active skin antenna," Smart Materials and Structures, vol. 26, article 025011, 2017.

[4] C. K. Kim, L. M. Lee, H. C. Park, W. Hwang, and W. S. Park, "Impact damage and antenna performance of conformal load-bearing antenna structures," Smart Materials and Structures, vol. 12, pp. 672-679, 2003.

[5] C. S. You and W. B. Hwang, "Design of load-bearing antenna structures by embedding technology of microstrip antenna in composite sandwich structure," Composite Structures, vol. 71, pp. 378-382, 2005.

[6] S. E. Morris, Y. Bayram, L. Zhang, Z. Wang, M. Shtein, and J. L. Volakis, "High-strength, metalized fibers for conformal load bearing antenna applications," IEEE Transactions on Antennas and Propagation, vol. 59, pp. 3458-3462, 2011.

[7] J. Zhou, J. Huang, L. Song, D. Zhang, and Y. Ma, "Electromechanical co-design and experiment of structurally integrated antenna," Smart Materials and Structures, vol. 24, article 037004, 2015.

[8] J. Zhou, L. Song, J. Huang, and C. Wang, "Performance of structurally integrated antennas subjected to dynamical loads," International Journal of Applied Electromagnetics and Mechanics, vol. 48, pp. 409-422, 2015.
[9] D. J. Hartl, G. J. Frank, G. H. Huff, and J. W. Baur, "A liquid metal-based structurally embedded vascular antenna: I. Concept and multiphysical modeling," Smart Materials and Structures, vol. 26, article 025001, 2017.

[10] D. J. Hartl, G. J. Frank, R. J. Malak, and J. W. Baur, "A liquid metal-based structurally embedded vascular antenna: II. Multiobjective and parameterized design exploration," Smart Materials and Structures, vol. 26, article 025002, 2017.

[11] L. Yao, X. Wang, F. J. Xu, D. Zhao, M. W. Jiang, and Y. P. Qiu, "Fabrication and impact performance of three-dimensionally integrated microstrip antennas with microstrip and coaxial feeding," Smart Materials and Structures, vol. 18, article 095034, 2009.

[12] L. Yao and Y. P. Qiu, "Design and fabrication of microstrip antennas integrated in three dimensional orthogonal woven composites," Composites Science and Technology, vol. 69, pp. 1004-1008, 2009.

[13] F. J. Xu, L. Yao, X. Wang, and Y. P. Qiu, "Effect of conductive yarn crimp in radiation patch on electromagnetic performance of 3D integrated microstrip antenna," Composites Part BEngineering, vol. 43, pp. 465-470, 2012.

[14] F. Xu, L. Yao, D. Zhao, L. Zhao, M. Jiang, and Y. Qiu, "Performance and impact damage of a three dimensionally integrated microstrip feeding antenna structure," Composite Structures, vol. 93, pp. 193-197, 2010.

[15] C. You, D. Kim, S. Cho, and W. Hwang, "Impact behavior of composite antenna array that is conformed around cylindrical bodies," Composites Science and Technology, vol. 70, pp. 627$632,2010$.

[16] D. Kim, C. You, and W. Hwang, "Effect of adhesive bonds on electrical performance in multi-layer composite antenna," Composite Structures, vol. 90, pp. 413-417, 2009.

[17] D. Kim, J. Kim, J. Kim et al., "Design and fabrication of a composite-antenna-structure for broadband frequency with microwave absorber," Journal of Composite Materials, vol. 46, pp. 1851-1858, 2012.

[18] W. C. Bing Zhang, W. Yanjie, K. Ding, and R. Li, "Review of 3D printed millimeter-wave and terahertz passive devices," International Journal of Antennas and Propagation, vol. 2017, Article ID 1297931, 10 pages, 2017.

[19] H. Xin and M. Liang, "3-D-printed microwave and $\mathrm{THz}$ devices using polymer jetting techniques," Proceedings of the IEEE, vol. 105, pp. 737-755, 2017.

[20] W. J. Otter and S. Lucyszyn, "Hybrid 3-D-printing technology for tunable $\mathrm{THz}$ applications," Proceedings of the IEEE, vol. 105, pp. 756-767, 2017.

[21] M. Liang, C. Shemelya, E. MacDonald, R. Wicker, and H. Xin, "3-D printed microwave patch antenna via fused deposition method and ultrasonic wire mesh embedding technique," IEEE Antennas and Wireless Propagation Letters, vol. 14, pp. 1346-1349, 2015.

[22] J. J. Adams, E. B. Duoss, T. F. Malkowski et al., "Conformal printing of electrically small antennas on three-dimensional surfaces," Advanced Materials, vol. 23, pp. 1335-1340, 2011.

[23] M. Liang, W. R. Ng, K. H. Chang, K. Gbele, M. E. Gehm, and H. Xin, "A 3-D Luneburg lens antenna fabricated by polymer jetting rapid prototyping," IEEE Transactions on Antennas and Propagation, vol. 62, pp. 1799-1807, 2014.

[24] P. Nayeri, M. Liang, R. A. Sabory-Garcia et al., "3D printed dielectric reflectarrays: low-cost high-gain antennas at sub- 
millimeter waves," IEEE Transactions on Antennas and Propagation, vol. 62, pp. 2000-2008, 2014.

[25] J. A. Byford, M. I. M. Ghazali, S. Karuppuswami, B. L. Wright, and P. Chahal, "Demonstration of RF and microwave passive circuits through 3-D printing and selective metalization," IEEE Transactions on Components Packaging and Manufacturing Technology, vol. 7, pp. 463-471, 2017.

[26] M. Y. Chen, D. Pham, H. Subbaraman, X. J. Lu, and R. T. Chen, "Conformal ink-jet printed C-band phasedarray antenna incorporating carbon nanotube field-effect transistor based reconfigurable true-time delay lines," IEEE Transactions on Microwave Theory and Techniques, vol. 60, pp. 179-184, 2012.

[27] H. Subbaraman, D. T. Pham, X. C. Xu et al., "Inkjet-printed two-dimensional phased-array antenna on a flexible substrate," IEEE Antennas and Wireless Propagation Letters, vol. 12, pp. 170-173, 2013.

[28] X. Lan, X. Lu, M. Y. Chen et al., "Direct on-Chip 3-D aerosol jet printing with high reliability," IEEE Transactions on Components, Packaging and Manufacturing Technology, vol. 7, no. 8, pp. 1369-1376, 2017.

[29] B. Shahriari, K. Swersky, Z. Y. Wang, R. P. Adams, and N. de Freitas, "Taking the human out of the loop: a review of Bayesian optimization," Proceedings of the IEEE, vol. 104, pp. 148-175, 2016.

[30] P. Chen, B. M. Merrick, and T. J. Brazil, "Bayesian optimization for broadband high-efficiency power amplifier designs," IEEE Transactions on Microwave Theory and Techniques, vol. 63, pp. 4263-4272, 2015.

[31] P. Chen, J. Xia, B. M. Merrick, and T. J. Brazil, "Multiobjective Bayesian optimization for active load modulation in a broadband 20-W GaN Doherty power amplifier design," IEEE Transactions on Microwave Theory and Techniques, vol. 65, pp. 860-871, 2017. 


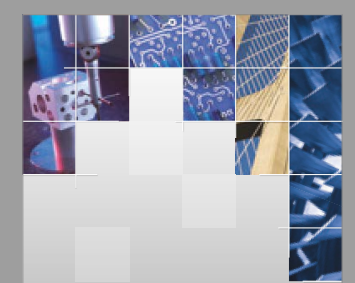

\section{Enfincering}
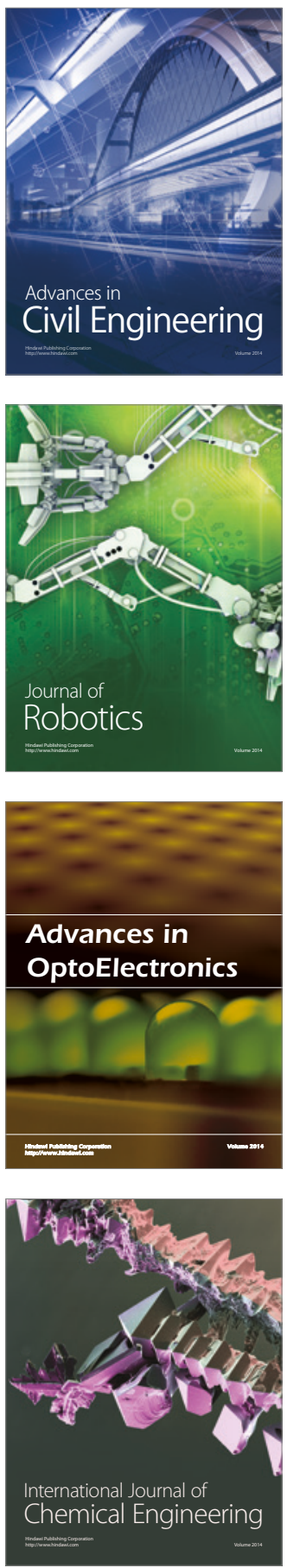

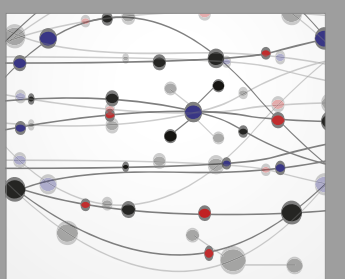

The Scientific World Journal

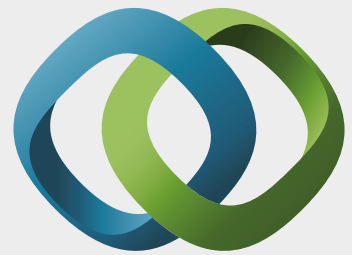

\section{Hindawi}

Submit your manuscripts at

https://www.hindawi.com
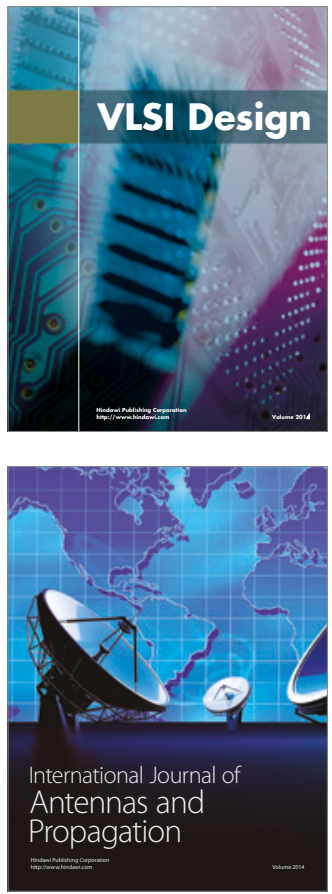

\section{Rotating}

Machinery
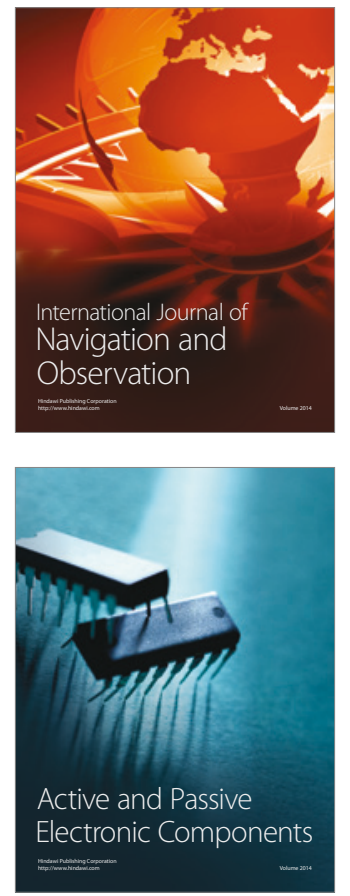
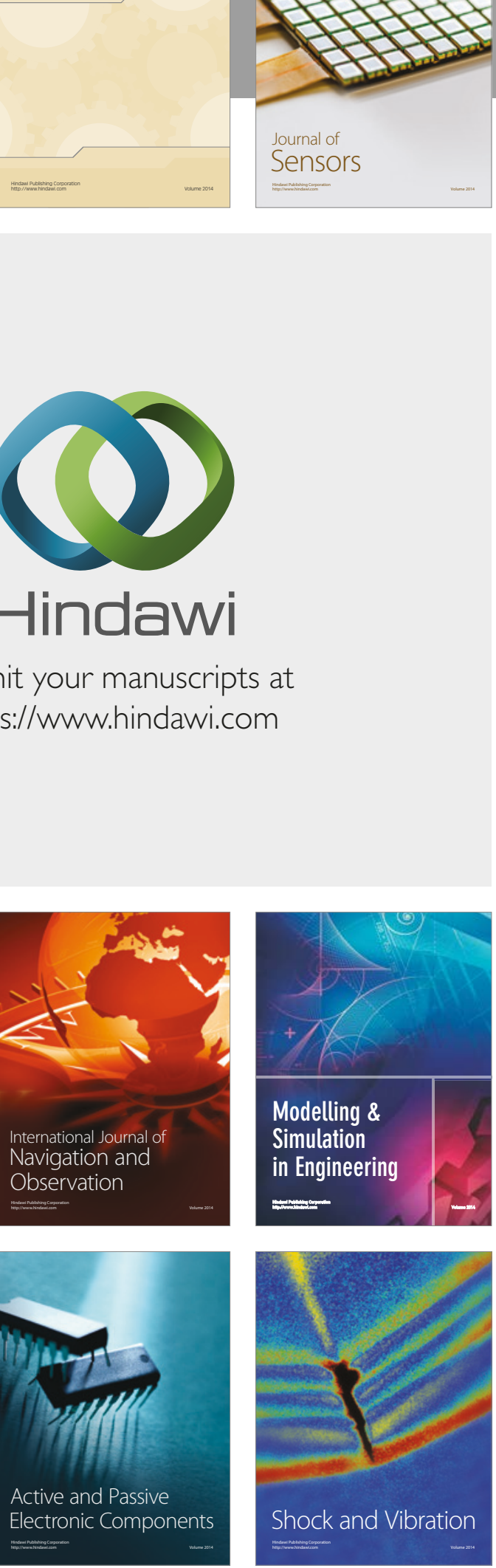
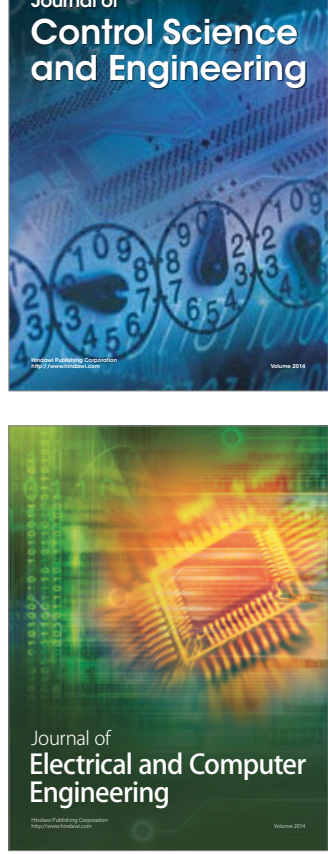

Distributed

Journal of

Control Science

and Engineering
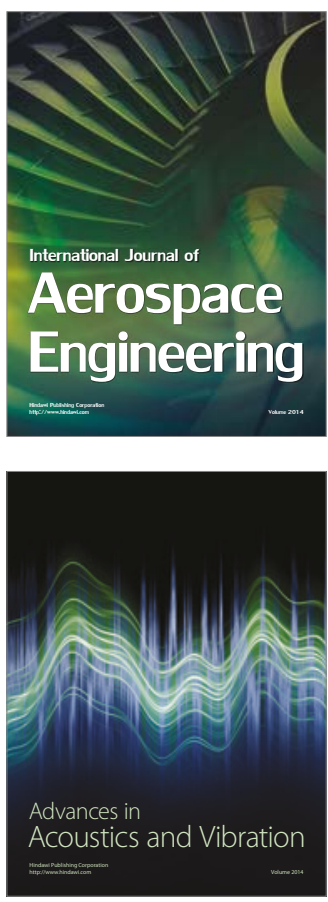

Sensor Networks 